OPEN ACCESS

Edited by:

Yundai Chen,

Chinese PLA General Hospital, China

Reviewed by:

Rayna Anderson,

University of Alabama in Huntsville,

United States

Anna Schmidt

Western Colorado University,

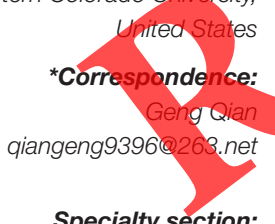

Specialty section:

This article was submitted to

Integrative Physiology,

a section of the journal

Frontiers in Physiology

Received: 30 July 2021

Accepted: 28 September 2021

Published: 28 October 2021

Citation:

Wu D, Kampmann E and Qian G (2021) Novel Insights Into the Role of Mitochondria-Derived Peptides

in Myocardial Infarction.

Front. Physiol. 12:750177.

doi: 10.3389/fphys.2021.750177

\section{Novel Insights Into the Role of Mitochondria-Derived Peptides in Myocardial Infarction}

\author{
Dan Wu', Enny Kampmann ${ }^{2}$ and Geng Qian ${ }^{1 *}$
}

1 Department of Cardiology, The First Medical Center, Chinese People's Liberation Army Hospital, Medical School of Chinese People's Liberation Army, Beijing, China, ${ }^{2}$ School of Life Sciences, City College of San Franciseo, San Francisco, CA, United States

Mitochondria-derived peptides (MDPs) are a new class of bioactive peptides encoded by small open reading frames (sORFs) within known mitochondrial DNA (mtDNA) genes. MDPs may affect the expression of nuclear genes and play cytoprotective roles against chronic and age-related diseases by maintaining mitochondrial function and cell viability in the face of metabolic stress and cyrotoxic insults. In this review, we summarize clinical and experimental findings indicating that MDPs act as local and systemic regulators of glucose homeostasis, immune and inflammatory responses, mitochondrial function, and adaptive stress responses, and focus on evidence supporting the protective effects of MDPs against myocardial infarction. These insights into MDPs actions suggest their potential in the treatment of cardiovascular diseases and should encourage further research in this field.

Keywords: myocardial infarction, mitochondria-derived peptides, mitochondria, mitochondria-ER communication, heart

\section{INTRODUCTION}

Mitochondria are semi-autonomous, double-membrane organelles that play critical roles in maintaining cellular homeostasis by governing cell energy metabolism and influencing signal transduction, reactive oxygen species (ROS)-mediated oxidative stress, and apoptosis (Brooks, 2018; Cao et al., 2020). Mitochondrial biogenesis is a highly dynamic process, and the rapid recycling and turnover of mitochondrial components enables these organelles to adapt to metabolic changes resulting from different cellular stressors (Del Campo, 2019; Chiang et al., 2020; Jin et al., 2021). Thus, a decline in mitochondrial function is frequently associated with numerous diseases. Extensive research sought to elucidate how mitochondria dysfunction affects the onset and progression of myocardial infarction (MI), a condition characterized by impaired ATP synthesis and energy metabolism, enhanced apoptosis, and abnormal $\mathrm{Ca}^{2+}$ dynamics in cardiac cells (Sommer et al., 2016; Daiber and Münzel, 2020; Fender et al., 2020; Gori et al., 2020).

Cellular homeostasis is critically regulated by the interaction between mitochondria and the cell nucleus via coordinated expression of mitochondrial and nuclear genes. Importantly, this genetic crosstalk also allows cells to cope with environmental and metabolic stress (Ryan and Hoogenraad, 2007; Quirós et al., 2016). The mitochondrial DNA (mtDNA) contains 37 genes that encode 13 polypeptides, all subunits of the electron transport chain (ETC) (Mangalhara and Shadel, 2018), as well as 2 ribosomal RNAs (rRNAs) and 22 transfer RNAs (tRNAs) that are required for their translation (Michel et al., 2015). In contrast with nuclear-encoded genes, the synthesis 
of mitochondrial proteins is independent of the translational machinery associated with the endoplasmic reticulum. Still, mtDNA-encoded proteins represent only $\sim 1 \%$ of the mitochondrial proteome, which is estimated at up to 1,500 proteins that are therefore predominantly encoded by nuclear DNA (Calvo and Mootha, 2010).

Mitochondria-derived peptides (MDPs) conform a new class of peptides encoded by small open reading frames (sORFs) within known mtDNA genes (Yen et al., 2013; Kim et al., 2017). MDPs are widely distributed in various tissues such as heart, vascular wall, kidney, skeletal muscle, and colon. MDPs were shown to affect the expression of nuclear genes and play cytoprotective roles through maintaining mitochondrial function and cell viability under both normal and pathological conditions (Krejcova et al., 2004; Tajima et al., 2005). In this review, we summarize the effects of MDPs on immune and inflammatory responses, glucose homeostasis, mitochondrial function, adaptive stress responses, and apoptosis, and discuss the protective actions of MDPs against MI (Mottis et al., 2019). We hope this information will stimulate further research to materialize the therapeutic potential of MDPs in the treatment of MI and cardiovascular disease.

\section{CLASSIFICATION AND FUNCTIONS OF MITOCHONDRIA-DERIVED PEPTIDES}

Eight MDPs have been identified up to date, all of them transcribed from sORFs harbored in mtDNA genes encoding $12 \mathrm{~S}$ rRNA and $16 \mathrm{~S}$ rRNA transcripts (Lee et al., 2015). The $12 \mathrm{~S}$ rRNA gene is 954 nucleotides long, extends from nucleotides 648 to 1,601 (representing about $6 \%$ of the full mtDNA), and the encoded product presents a conserved secondary structure. The 16S rRNA gene is 1,559 nucleotides long and extends between nucleotides 1,671-3,229 of the mtDNA (Galtiey et al., 2006). Several studies confirmed that MDPs regulate cellular metabolism and survival by maintaining glucose homeostasis, antioxidant capacity, and antiapoptotic signaling by binding to intracellular and extracellular receptors through autocrine and paracrine mechanisms (Yang et al., 2019).

\section{Humanin}

Humanin (HN), the first discovered MDP, was identified in the brain of a patient with Alzheimer's disease (AD) by Hashimoto and his team in 2001 (Hashimoto et al., 2001). Through different translational machineries, two HN peptides, 21 and 24 amino acids long, are produced, respectively, in mitochondrial and cytoplasmic compartments from a sORF located in the 16S rRNA gene of mtDNA (Sreekumar and Kannan, 2020). HN is found in circulating body fluids, such as blood and cerebrospinal fluid, and in metabolically active organs such as the heart, liver, and kidneys (Arakawa et al., 2008; Muzumdar et al., 2009; Chin et al., 2013). In addition, nuclear DNA contains several ORF sites, highly homologous to the mtDNA sequence encoding $\mathrm{HN}$, which potentially give rise to several HN-like peptides (Jiang et al., 2020; Jusic and Devaux, 2020).
Humanin and its synthetic analogs have been shown to have significant cytoprotective and glucose-lowering effects. For example, the HN analog S14G (HNG) produced by substitution of serine by glycine at position 14 in $\mathrm{HN}$, is 1,000 times more potent than HN (Arakawa et al., 2008). In turn, the HN homolog HNGF6A, formed by an additional substitution of phenylalanine by alanine at position 6 , was shown to have an even greater effect than HN and HNG in improving central insulin sensitivity and lowering blood glucose levels in diabetic rats by counteracting the proapoptotic actions of insulin like growth factor binding protein-3 (IGFBP-3) (Muzumdar et al., 2009). Indeed, extensive research demonstrated that $\mathrm{HN}$ plays a protective role against various pathological conditions, including neurodegenerative diseases (Cui et al., 2017), diabetes (Xie et al., 2014), endothelial dysfunction (Ding et al., 2019), and cardiovascular disease (Ren et al., 2020).

\section{Apoptosis}

The antiapoptotic action of HN has been shown to result from inhibition of Bax-induced pore formation in the mitochondrial outer membrane and subsequent suppression of cytochrome $\mathrm{c}$ release ( $\mathrm{Ma}$ and Liu, 2018). Besides. $\mathrm{HN}$ was shown to engage the Bid BH3 domain, which mediates the association of Bid with other Bcl-2 family members (Choi et al., 2007), and to bind directly to the extra-long isoform of Bim (BimEL). The ensuing inhibition of BimEL may thus contribute to the antiapoptotic properties of HN (Luciano et al., 2005). The therapeutic potential of $\mathrm{HN}$ as an antiapoptotic agent has been confirmed by in vitro and in vivo experiments demonstrating increased neuroprotection after HN binding to IGFBP-3 (Lee et al., 2013).

\section{Oxidative Stress}

Recent research also unveiled significant antioxidant properties for $\mathrm{HN}$ and its derivatives. In cardiac myoblasts challenged with $\mathrm{H}_{2} \mathrm{O}_{2}$, exposure to $\mathrm{HNG}$ lowered ROS levels, preserved mitochondrial membrane potential and ATP levels, induced activation of catalase and glutathione peroxidase, and decreased the ratio of oxidized to reduced glutathione (GSH) (Klein et al., 2013). Along these lines, HNG showed to beneficially regulate GSH and sphingolipid metabolism in a rat model of dietinduced obesity [46].

\section{Inflammation}

Zhao et al. (2013) showed that pretreatment with HN decreased the secretion of proinflammatory cytokines, i.e., interleukin (IL)-6, IL-1 $\beta$, and tumor necrosis factor $\alpha(\mathrm{TNF} \alpha)$, induced by lipopolysaccharide (LPS) in cultured astrocytes. In turn, Jung et al. (2020) reported that intravenously administered HN promoted a "reparative" microglia phenotype characterized by enhanced phagocytosis and reduced proinflammatory responses in a mouse model of intracerebral hemorrhage.

\section{Regulation of Mitochondrial Function}

Many studies have examined the role of $\mathrm{HN}$ in the regulation of mitochondrial homeostasis. Experiments in human retinal pigment epithelial cells showed that $\mathrm{HN}$ exposure preserved essential functions related to energy production by increasing basal oxygen consumption rate, maximum respiration rate, 
respiration capacity, and ATP production (Sreekumar et al., 2016; Kleinbongard, 2020; Lahiri et al., 2020; Lindner et al., 2020). In pancreatic MIN6 $\beta$-cells, HN promoted mitochondrial biogenesis by increasing mitochondrial mass, mtDNA copy number, and PGC-1 $\alpha$, NRF1, and mitochondrial transcription factor A (mtTFA) levels (Qin et al., 2018b). Similarly, the HN analog HNG counteracted oxidative stress-induced mitochondrial dysfunction in cardiac tissue by reducing ROS generation and stabilizing mitochondrial membrane potential, mitochondrial structure, and ATP levels (Klein et al., 2013; Lobo-Gonzalez et al., 2020; Lyu et al., 2020; Sawashita et al., 2020). However, further research is needed to clarify the specific mechanisms by which HN sustains mitochondrial integrity and function in mammalian cells.

\section{MOTS-c}

Mitochondrial open reading frame of the $12 \mathrm{~S}$ rRNA-c (MOTSc) is a 16-amino-acid peptide encoded by a sORF within the mitochondrial $12 \mathrm{~S}$ rRNA. Originally identified by Lee et al. (2015) in various tissues in rodents, as well as in human plasma, MOTSc proved to be an effective regulator of insulin sensitivity and metabolic homeostasis by inducing activation of AMP-activated protein kinase (AMPK). Subsequently, Kim et al. showed that MOTS-c can translocate to the nucleus in response to metabolic or oxidative stress, suggesting a novel role for MOTS-c in gene expression regulation via retrograde (mitochondria to nucleus) signaling (Quirós et al., 2016). Additional reports in animal models pointed out that MOTS-c regulates insulin resistance and attenuates the symptoms of hyperinsulinemia, obesity, and osteoporosis (Lee et al., 2015, 2016; Ming et al., 2016; Hu and Chen, 2018; Qin et al., 2018a; Lu et al., 2019; Mehta et al., 2019; Yan et al., 2019; Weng et al., 2021). Moreover, MOTS-c was shown to possess significant anti-inflammatory actions by inhibiting the expression of immune-related genes (Zhai et al, 2017; Li et al., 2018; Yan et al., 2019). Recently, Jiang et al. (2021) reported that MOTS-c administration enhanced object and location recognition memory formation and consolidation in mice treated with amyloid-beta peptide $(\mathrm{A} \beta 1-42)$ or LPS through activation of hippocampal AMPK

\section{Small Humanin-Like Peptides (SHLPs)}

Small humanin-like peptides (SHLP1-6) are encoded and translated from a sORF contained within the same 16S RNA gene harboring the HN-encoding sORF (Cobb et al., 2016). Fist identified by Cobb et al. (2016), the 20-38 amino-acidlong SHLP peptides were shown to be expressed in mouse tissues including heart, liver, brain, kidney, spleen, prostate, testis, and skeletal muscle. Using RT-PCR, a mitochondrial origin was established for SHLPs 1, 4, 5, and 6, whereas SHLP2 and SHLP3 were amplified from both mitochondrial and nuclear cDNA (Cobb et al., 2016). These authors also found that circulating SHLP2 levels decline with age, and that male mice had higher SHLP2 levels than female mice in both the young and old groups. These results indicated that SHLP2 secretion levels vary with age and sex.

The Cobb et al. (2016) study showed that similar to $\mathrm{HN}$, the neuroprotective actions of SHLP2 were associated with phosphorylation of both extracellular signal-regulated kinase
(ERK) and signal transducer and activator of transcription 3 (STAT-3). Concordant also with HN effects, both SHLP2 and SHLP3 were shown to improve mitochondrial quality control, enhance oxidant consumption rate, mitochondrial biogenesis, and ATP synthesis, and mediate anti-apoptotic effects (Cobb et al., 2016). The beneficial influence of SHLP2 and SHLP3 on age-related neurodegenerative disease is supported by evidence that both SHLP2 and SHLP3 improved neuronal survival following toxic insults (Cobb et al., 2016). In particular, a prominent antiapoptotic effect was revealed for SHLP2 both in neurons treated with A $\beta 1-42$ (Cobb et al., 2016) and in agerelated macular degeneration (AMD) cybrid cells containing mtDNA from AMD patients [REF]. Several studies reported additional roles for SHLPs in the modulation of cardiovascular function, insulin sensitization, inflammation, and GSH and sphingolipid metabolism [46]. Interestingly, low circulating levels of SHLP2 were linked with increased risk of prostate cancer (Xiao et al., 2017; Mehta et 1., 2019). Although these findings consistently affirm the beneficial actions of SHLPs on human health, further work is needed to elucidate the specific mechanisms mediating SHLPs' effects.

\section{PROTECTIVE ACTIONS OF MITOCHONDRIA-DERIVED PEPTIDES AGAINST RISK EACTORS FOR MYOCARDIAL INFARCTION}

Ischemic heart disease is the leading cause of morbidity and mortality in the world (Reed et al., 2017; Qiao et al., 2021; Wischmann et al., 2020; Yang Y. et al., 2020; Zhang L. et al., 2020). It develops as a consequence of risk factors such as systemic arterial hypertension, left ventricular (LV) hypertrophy, hyperlipidemia, atherosclerosis, insulin resistance, diabetes, and aging (Ollauri-Ibáñez et al., 2020; Wang et al., 2020e; Watson et al., 2020; Winter et al., 2020). Indeed, age represents the largest risk factor for cardiovascular diseases, including cardiac fibrosis, atrial fibrillation, and heart failure (Steenman and Lande, 2017; Santosa et al., 2020; Schinner et al., 2020; Seano and Jain, 2020; Selvaraju et al., 2020). As mentioned, a correlation between MDP expression and age-related diseases is suggested by the significant decline in circulating MDP levels that occurs with age (Cobb et al., 2016). Since oxidative stress and mitochondrial dysfunction are tightly involved in the mechanisms of age-related diseases, MDPs, especially HN and MOTS-c, the most studied ones, have emerged as promising therapeutic targets to treat neurological, cardiovascular, and metabolic conditions associated with advanced age (Zapała et al., 2010; Thummasorn et al., 2017; Yang et al., 2019; Kim et al., 2021).

Mitochondria-derived peptides were shown to critically influence lipid and glucose metabolism, two aspects closely related with myocardial disfunction and infarction. Cobb et al. (2016) reported that SHLP2 and SHLP3 enhanced 3T3-L1 preadipocyte differentiation and increased leptin levels in mice. Meanwhile increased peripheral glucose uptake and suppressed hepatic glucose production were observed after intracerebral 
infusion of SHLP2 in rats subjected to systemic pancreatic insulin clamp and physiologic hyperinsulinemic-euglycemic clamp (Cobb et al., 2016). This suggests that central activity of SHLP2 has obvious peripheral effects. Gong et al. (2015) showed that intraperitoneal administration of the HN homolog HNG can reduce weight, visceral fat contents, and hepatic steatosis in highfat diet (HFD)-fed mice. It is unclear, however, whether reduced adipogenesis or increased lipolysis mediated these effects. Lee et al. reported that MOTS-c enhanced lipid oxidation and glucose metabolism in skeletal muscle by increasing the expression of GLUT4 and inhibiting the folate-methionine cycle. The ensuing inhibition of de novo purine synthesis caused accumulation of endogenous 5-aminoimidazole-4-carboxamide ribonucleoside (AICAR), a purine precursor, leading to activation of AMPK. Because de novo purine synthesis is subjected to feedback regulation by purine nucleotides, MOTS-c was proposed to accelerate de novo purine synthesis, which is consistent with the observed increase in $\mathrm{NAD}^{+}$levels, glycolytic fluxes, and increased routing of glucose to the pentose phosphate pathway (PPP). Following AICAR accumulation, AMPK activation stimulates fatty acid oxidation via phosphorylation-induced inactivation of acetyl-CoA carboxylase (ACC) (Steinberg and Kemp, 2009). Studies from Lee et al. (2015) also showed that compared with control cells, HEK293 cells stably overexpressing MOTS-c exhibited higher levels of carnitine shuttles, reduced essential fatty acid levels, and increased levels of the $\beta$-oxidation intermediate myristoyl-CoA. These studies concluded that MOTS-c treatment prevented age-dependent and HFD-induced insulin resistance by enhancing GLUT4 expression and the rate of insulin-induced glucose utilization in skeletal muscle, without changes in the hepatic glucose production rate (Lee et al., 2015). In turn, Muzumdar et al. (2009) reported that intracerebroventricular administration of HN during pancreaticeuglycemic clamp increased insulin sensitivity, leading to a reduction in hepatic glucose production by inducing fatty acid metabolism and Akt signaling. Along these lines, Kuliawat et al. (2013) showed that glucose-stimulated insulin secretion was potently stimulated by the HN analog HNGF6A both in $\beta$ TC3 cells and in pancreatic islets from normal and diabetic mice.

Coronary artery atherosclerosis, characterized by lipid deposition, foam cell formation, and accumulation of cholesterol in the arterial wall, is the leading cause of myocardial ischemia and coronary artery disease (Tabas et al., 2007; Lyu et al., 2015; Sawashita et al., 2020; Zhang Y. et al., 2020; Zhao et al., 2020). Excessive production of ROS, caused by long-term occlusion of the coronary artery, enhances oxidative stress and inflammation, resulting in vascular endothelial dysfunction and accelerated formation of atherosclerotic plaques (Lu et al., 2020; Watanabe et al., 2020; Wincewicz and Woltanowski, 2020; Yang Q. K. et al., 2020). The expression of $\mathrm{HN}$ in the endothelial cell layer of human arteries and veins was first reported by Bachar and colleagues. They showed, through in vitro experiments, that $\mathrm{HN}$ protected against atherosclerosis by reducing ROS production and attenuating oxidative stress (Bachar et al., 2010). The formation of foam cells results from imbalanced cholesterol influx and efflux in arterial wall-associated macrophages and contributes to the onset and development of atherosclerosis.
Using cultured RAW 264.7 macrophages, Zhu et al. showed that HNG prevents ox-LDL-induced foam cell formation. This effect resulted from inhibition of CD36 and low-density lipoprotein receptor (LOX)-1 upregulation, which reduced ox-LDL endocytosis, coupled with upregulation of ATP-binding cassette (ABC) transporter A1 and ABCG1 levels, which enhanced ox-LDL efflux (Zhu et al., 2017). Hyperglycemia is an important contributor to the pathological development of atherosclerosis in diabetic patients. Wang et al. reported that $\mathrm{HN}$ treatment prevented high glucose-induced attachment of monocytes to human umbilical vein endothelial cells (HUVECs). This effect was mediated by ERK5 phosphorylation and induction of Krüppel-like factor 2 (KLF2) expression, upregulation of KLF2 target genes such as endothelial nitric oxide synthase (eNOs) and endothelin-1 (ET-1), and reduced expression of leukocyte adhesion molecules (VCAM-1 and E-selectin) (Wang et al., 2018). Qin et al. found that circulating MOTS-c levels were downregulated in patients with coronary endothelial dysfunction. They showed that plasma MOTS-c levels were positively correlated with microvascular and epicardial coronary endothelial function in study subjects, demonstrating also that exposure to MOTS-c had no direct vasoactive effects but improved acetylcholine-induced vasodilation in aortic explants from renal artery stenosis mice (Qinet al., 2018a).

\section{Role of Mitochondria-Derived Peptides in Myocardial Ischemic Injury and Ischemia/Reperfusion Injury}

A pivotal feature of ischemia is the inadequate supply of oxygen to the mitochondria to support oxidative phosphorylation (OXPHOS). This causes excessive ROS production and oxidative stress injury, leading to myocardial cell death (Zhu et al., 2018; Zhou et al., 2019; Hughes et al., 2020; Wang et al., 2020c). Myocardial ischemic injury usually results in infarction, arrhythmias, and decreased myocardial contractility. Reperfusion therapy refers to procedures that allow the rapid return of blood flow to the ischemic area of the myocardium, through which mortality can be approximately halved (Jin et al., 2018; Smadja et al., 2020; Tan et al., 2020; Zhu et al., 2021). However, reperfusion itself may induce irreversible cell injury (e.g., necrosis and apoptosis), thus leading to extensive infarct size, diminished cardiac contractile function, and arrhythmia (Zhou et al., 2017a,b, 2018c; Szulcek et al., 2020; Wang et al., $2020 \mathrm{~d})$. The ensuing reconstruction of the damaged myocardium poses a big clinical challenge, as it is a key contributor to cardiac dysfunction after MI (Zhou et al., 2018b, 2020; Singh et al., 2020; Wang et al., 2020a). Myocardial fibrosis represents a secondary response to the pathophysiologic remodeling process. It involves profound changes in the interstitial myocardial collagen network, facilitating the development of cardiac dysfunction and arrhythmias and influencing the clinical course and outcome of heart failure patients (Zhou et al., 2018a; Tomita et al., 2020; Wang et al., 2020b).

Myocardial injury begins after about 20 min of coronary occlusion, first affecting the subendocardium and papillary 
muscle, and extending thereafter into the mid-myocardial bedat-risk by about 60-90 min (Reimer et al., 1977). Myocardial injury may be reversible because of the activation of physiologic adaptations manifested in myocardial stunning, hibernation, and pre- and post-ischemic conditioning (Heyndrickx et al., 1975). However, prolonged ischemia causes irreversible myocardial injury regardless of the tissue's hypoxic tolerance and intrinsic adaptive mechanisms. In this setting, oxygen consumption by oxidative phosphorylation and the synthesis of high energy phosphate products are reduced, along with decreased availability of malate (a complex I substrate) and succinate (a complex II substrate) in mitochondria (McCully et al., 2007). Alterations in complex I activity and accumulation of succinate during ischemia may be related to mitochondrial oxidative injury during reperfusion (Pacher et al., 2006). Here, the maximal rate of hydrolyzed succinate overwhelms the speed of ATP synthesis, leading to a phenomenon called reverse electron transport (RET) that results in enhanced ROS production by complex I. Accordingly, pharmacological inhibition of complex I slows the reactivation of mitochondria and reduces ROS (Chouchani et al., 2013). Multiple biochemical and ultrastructural changes occur in cardiac cells upon ischemia-induced ATP depletion. Faced with oxygen deprivation, the heart switches from fatty acid oxidation to anaerobic glycolysis to sustain ATP production, leading to the accumulation of lactate and a decline in cellular pH (Walker et al., 2000; Khabbaz et al., 2001). Intracellular acidification stimulates the activity of the $\mathrm{Na}^{+} / \mathrm{H}^{+}$exchanger, which enhances in turn $\mathrm{Ca}^{2+}$ influx by activating the $\mathrm{Na}^{+} / \mathrm{Ca}^{2+}$ exchanger as a way to remove excess $\mathrm{Na}^{+}$into the extracellular space. This leads eventually to mitochondrial $\mathrm{Ca}^{2+}$ overload, which ultimately results in mitochondrial swelling, increased mitochondrial intermembrane distance, and deficient OXPHOS (Pagliaro and Penna, 2015).

As with myocardial ischemic ischemia/reperfusion (I/R) injury varies based upon reversible events, onset of reperfusion arrhythmias, cardiac stunning, etc., which determine the eventual occurrence of lethal reperfusion injury (Hausenloy et al., 2016). Clinically, $\mathrm{E} / \mathrm{R}$ injury is associated with the disruption of the microvasculature leading to the noreflow phenomenon and activation of inflammatory reactions (Wang et al., 2020b). Over the last decades, abundant research focusing on lethal myocardial reperfusion injury reported the mechanisms involved in this process. These alterations include rapid normalization of $\mathrm{pH}$, intracellular $\mathrm{Ca}^{2+}$ overload, and $\mathrm{ROS}$ generation, all of which aggravate mitochondrial dysfunction (Pan et al., 2013). A hallmark of the latter is the opening of the mitochondrial permeability transition pore (mPTP), which is thought to be the most noxious step during reperfusion injury, leading to activation of apoptotic and necrotic signaling pathways (Ruiz-Meana et al., 2009).

Several studies suggested that $\mathrm{HN}$ or its synthetic analogs might be effective to treat MI. Recently, Wijenayake et al. uncovered the cytoprotective role of a humanin homolog (TSEhumanin), expressed in freshwater turtles, against sustained hypoxia and oxidative damage (Wijenayake and Storey, 2021). In a rat model of myocardial $\mathrm{I} / \mathrm{R}$ injury, Thummasorn et al. showed that endogenous HN levels were decreased at the end of cardiac I/R. Interestingly, intravenous injection of $\mathrm{HNG}$ $15 \mathrm{~min}$ before I/R (but not during I/R) significantly decreased arrhythmia incidence and infarct size, improved cardiac mitochondrial function, and attenuated cardiac dysfunction. The same group later showed that high- dose HNG $(252 \mu \mathrm{g} / \mathrm{kg})$ administration during the ischemic phase increased myocardial $\mathrm{HN}$ levels, reduced arrhythmia, myocardial infarction area, and mitochondrial dysfunction. These effects were associated with AKT signaling activation, inhibition of Bax translocation to the mitochondrial membrane, and apoptosis prevention (Thummasorn et al., 2017). Subsequently, using isolated cardiac mitochondria, the same group showed that HNG was more effective than cyclosporine $\mathrm{A}$ in decreasing oxidative stress and alleviated mitochondrial damage caused by $\mathrm{H}_{2} \mathrm{O}_{2}$ by decreasing complex I activity (Thummasorn et al., 2018). Similarly, Muzumdar et al. showed that administration of HNG one hour before or at the time of reperfusion improved LV function and decreased infarct size in a mouse model of $\mathrm{I} / \mathrm{R}$. The suggested mechanism involved AMPK/eNOS signaling and downregulation of pro-apoptotic factors (Muzumdar et al., 2010)

\section{Role of Mitochondria-Derived Peptides in Post-infarction Cardiac Fibrosis}

The development of post-MI heart failure is associated with complex and progressive cellular and ultrastructural transformation eyents resulting in ventricular remodeling, a phenomenon first described by Tennant and Wiggers in the 1930s (Tennant and Wiggers, 1935). The human left ventricle has 2 to 4 billion cardiomyocytes, and a MI can cause the death of $\geq 25 \%$ of this population in a few hours (Beltrami al., 2001). Due to the heart's limited ability for rapid selfrepairing after catastrophic damage, scar formation, rather than muscle regeneration, is often the major component of the healing response following MI (Olivetti et al., 1991). The mechanisms of post-infarction cardiac remodeling include interactions between cellular, extracellular, and neurohormonal components. Early changes occurring within the first $72 \mathrm{~h}$ of an acute myocardial insult include expansion of the infarct zone, mainly because of the degradation of intermyocyte collagen struts by serine proteases and activated matrix metalloproteinases (MMPs) released from neutrophils. Concomitantly, myocardial necrosis determines an influx of inflammatory cells, including macrophages and other antigen-presenting cells, which results in wall thinning, ventricular dilatation, and eventually, cardiac rupture (Sutton and Sharpe, 2000). Late remodeling mainly involves eccentric hypertrophy and LV cavity dilation because of the increased load on the non-infarcted myocardium (Anversa et al., 1985). Adverse cardiac remodeling is facilitated by the imbalance between MMPs and their inhibitors (tissue inhibitors of metalloproteinases; TIMPs). These are regulated by several transcription factors and enzymes, including the NF- $\mathrm{B}$ and JAK-STAT pathways, which are influenced by the renin-angiotensin-aldosterone system (RAAS) (Chen et al., 2004). Despite the extensive knowledge accumulated so far, clinical treatment of post-MI heart failure is still very challenging and thus requires further research. 
Contrasting with the solid preclinical evidence supporting the beneficial actions of $\mathrm{HN}$ and its analogs in cardiac I/R injury, research on the possible influence of MDPs in cardiac remodeling remains scarce. Recently, Wei et al. (2020) reported that treatment with MOTS-c significantly decreased blood pressure, maintained normal cardiac structure, reversed ventricular remodeling, and reduced the stiffness of blood vessels in a rat model of vascular calcification induced by vitamin D3 plus nicotine (VDN) treatment. They further showed that MOTSc attenuated VDN-induced vascular calcification pathology by stimulating AMPK signaling, reversing also the upregulation of angiotensin II type 1 (AT-1) and endothelin B (ET-B) receptors mediated by VDN. Overexpression of AT-1 receptors is linked to increased myocardial fibrosis and cardiac dysfunction, which is consistent with the beneficial effect of MOTS-c against both oxidative stress and development of myocardial contractile dysfunction (Honda et al., 2018). Regarding ET-B, experiments with the AMPK agonist AICAR indicated that AMPK activation downregulates ET-B receptor expression, stimulates autophagy, and normalizes contractile responses to the ET-B agonist sarafotoxin $6 \mathrm{c}$ in VSMCs cultured under high glucose conditions (Chen et al., 2018).

\section{REFERENCES}

Anversa, P., Beghi, C., Kikkawa, Y., and Olivetti, G. (1985). Myocardial response to infarction in the rat. Morphometric measurement of infarct size and myocyte cellular hypertrophy. Am. J. Pathol. 118, 484-492.

Arakawa, T., Kita, Y., and Niikura, T. (2008). A rescue factor for Alzheimer's diseases: discovery, activity, structure, and mechanism. Curr. Med. Chem. 15, 2086-2098. doi: 10.2174/092986708785747616

Bachar, A. R., Scheffer, L., Schroeder, A. S., Nakamura, H. K. Cobb, L. J., Oh, Y. K., et al. (2010). Humanin is expressed in human vaseular walls and has a cytoprotective effect against oxidized LDL-induced oxidative stress. Cardiovasc. Res. 88, 360-366. doi: 10.1093/cvr/cvq191

Beltrami, A. P., Urbanek, K., Kajstura, J., R., et al. (2001). Evidence that human cardiac myocytes divide after myocardial infarction. N. Engl. J. Med. 344, 1750-1757. doi: 10.1056/ nejm200106073442303

Brooks, H. R. (2018). Mitochondria: finding the power to change. Cell 175, 891-893. doi: 10.1016/j.cell.2018.10.03

Calvo, S. E., and Mootha, V. K. (2010). The mitochondrial proteome and human disease. Annu. Rev. Genomics Hum. Genet. 11, 25-44. doi: 10.1146/annurevgenom-082509-141720

Cao, F., Maguire, M. L., McAndrew, D. J., Lake, H. A., Neubauer, S., Zervou, S., et al. (2020). Overexpression of mitochondrial creatine kinase preserves cardiac energetics without ameliorating murine chronic heart failure. Basic Res. Cardiol. 115:12. doi: 10.1007/s00395-020-0777-3

Chen, K., Chen, J., Li, D., Zhang, X., and Mehta, J. L. (2004). Angiotensin II regulation of collagen type I expression in cardiac fibroblasts: modulation by PPAR-gamma ligand pioglitazone. Hypertension 44, 655-661. doi: 10.1161/01. HYP.0000144400.49062.6b

Chen, Y., Zhang, H., Liu, H., Li, K., Jia, M., and Su, X. (2018). High glucose upregulated vascular smooth muscle endothelin subtype B receptors via inhibition of autophagy in rat superior mesenteric arteries. Ann. Vasc. Surg. 52, 207-215. doi: 10.1016/j.avsg.2018.02.028

Chiang, J. L., Shukla, P., Pagidas, K., Ahmed, N. S., Karri, S., Gunn, D. D., et al. (2020). Mitochondria in ovarian aging and reproductive longevity. Ageing Res. Rev. 63:101168. doi: 10.1016/j.arr.2020.101168

Chin, Y. P., Keni, J., Wan, J., Mehta, H., Anene, F., Jia, Y., et al. (2013). Pharmacokinetics and tissue distribution of humanin and its analogues in male rodents. Endocrinology 154, 3739-3744.

\section{OUTLOOK AND PERSPECTIVES}

Mitochondrial dysfunction is closely correlated with the symptoms of MI and cardiovascular disease (Suárez-Rivero et al., 2016). Available data suggest that impaired synthesis of MDPs in cardiomyocytes and endothelial cells contributes to the pathological sequelae of cardiac I/R injury. Accordingly, treatment with MDPs was shown to alleviate ischemic injury, limit infarct area, and attenuate adverse cardiac remodeling after experimental infarction in rodent models. However, in the setting of $\mathrm{MI}$, addressing the optimal time window at which MDPs exert maximal effects would help validate their use as pre-, per-, and/or post-conditioning agents. Collectively, the findings summarized above suggest the therapeutic potential of MDPs to treat MI as well as other common age-related diseases.

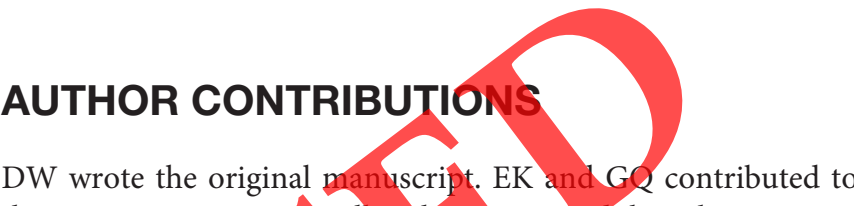
the manuscript revision. All authors approyed the submission.

Choi, J., Zhai, D, Zhou, X., Satterthwait, A., Reed, J. C., and Marassi, F. M. (2007). Mapping the specific cytoprotective interaction of humanin with the pro-apoptotic protein bid. Chem. Biol. Drug Des. 70, 383-392. doi: 10.1111/j. 1747-0285.2007.00576.x

Chouchani, E. T., Methner, C., Nadtochiy, S. M., Logan, A., Pell, V. R., Ding, S., et al. (2013) Cardioprotection by S-nitrosation of a cysteine switch mitochondrial complex I. Nat. Med. 19, 753-759. doi: 10.1038/nm. 3212

Cobb, L. J., Lee, C., Xiao, J., Yen, K., Wong, R. G., Nakamura, H. K., et al. (2016). Naturally occurring mitochondrial-derived peptides are age-dependent regulators of apoptosis, insulin sensitivity, and inflammatory markers. Aging 8, 796-809. doi: 10.18632/aging.100943

Cui, A. L., Zhang, Y. H., Li, J. Z., Song, T., Liu, X. M., Wang, H., et al. (2017). Humanin rescues cultured rat cortical neurons from NMDA-induced toxicity through the alleviation of mitochondrial dysfunction. Drug Des. Dev. Ther. 11, 1243-1253. doi: 10.2147/dddt.S133042

Daiber, A., and Münzel, T. (2020). Interplay of the red blood cell and vascular endothelial nitric oxide synthase system to combat cardiac complications of anemia. Basic Res. Cardiol. 115:44. doi: 10.1007/s00395-020-0 801-7

Del Campo, A. (2019). Mitophagy as a new therapeutic target for sarcopenia. Acta Physiol. 225:e13219. doi: 10.1111/apha.13219

Ding, Y., Feng, Y., Zhu, W., Zou, Y., Xie, Y., Wang, F., et al. (2019). [Gly14]Humanin Prevents Lipid Deposition and Endothelial Cell Apoptosis in a Lectin-like Oxidized Low-density Lipoprotein Receptor-1-Dependent Manner. Lipids 54, 697-705. doi: 10.1002/lipd.12195

Fender, A. C., Kleeschulte, S., Stolte, S., Leineweber, K., Kamler, M., Bode, J., et al. (2020). Thrombin receptor PAR4 drives canonical NLRP3 inflammasome signaling in the heart. Basic Res. Cardiol. 115:10. doi: 10.1007/s00395-0190771-9

Galtier, N., Enard, D., Radondy, Y., Bazin, E., and Belkhir, K. (2006). Mutation hot spots in mammalian mitochondrial DNA. Genome Res. 16, 215-222. doi: 10.1101/gr.4305906

Gong, Z., Su, K., Cui, L., Tas, E., Zhang, T., Dong, H. H., et al. (2015). Central effects of humanin on hepatic triglyceride secretion. Am. J. Physiol. Endocrinol. Metab. 309, E283-E292. doi: 10.1152/ajpendo.00043.2015

Gori, T., Lelieveld, J., and Münzel, T. (2020). Perspective: cardiovascular disease and the Covid-19 pandemic. Basic Res. Cardiol. 115:32. doi: 10.1007/s00395020-0792-4 
Hashimoto, Y., Niikura, T., Ito, Y., Sudo, H., Hata, M., Arakawa, E., et al. (2001). Detailed characterization of neuroprotection by a rescue factor humanin against various Alzheimer's disease-relevant insults. J. Neurosci. 21, 9235-9245. doi: 10.1523/jneurosci.21-23-09235.2001

Hausenloy, D. J., Barrabes, J. A., Bøtker, H. E., Davidson, S. M., Di Lisa, F., Downey, J., et al. (2016). Ischaemic conditioning and targeting reperfusion injury: a 30 year voyage of discovery. Basic Res. Cardiol. 111:70. doi: 10.1007/s00395-0160588-8

Heyndrickx, G. R., Millard, R. W., McRitchie, R. J., Maroko, P. R., and Vatner, S. F. (1975). Regional myocardial functional and electrophysiological alterations after brief coronary artery occlusion in conscious dogs. J. Clin. Invest. 56, 978-985. doi: 10.1172/jci108178

Honda, J., Kimura, T., Sakai, S., Maruyama, H., Tajiri, K., Murakoshi, N., et al. (2018). The glucagon-like peptide-1 receptor agonist liraglutide improves hypoxia-induced pulmonary hypertension in mice partly via normalization of reduced ET(B) receptor expression. Physiol. Res. 67(Suppl. 1), S175-S184. doi: $10.33549 /$ physiolres.933822

Hu, B. T., and Chen, W. Z. (2018). MOTS-c improves osteoporosis by promoting osteogenic differentiation of bone marrow mesenchymal stem cells via TGFB/Smad pathway. Eur. Rev. Med. Pharmacol. Sci. 22, 7156-7163. doi: 10.26355/ eurrev_201811_16247

Hughes, W. E., Beyer, A. M., and Gutterman, D. D. (2020). Vascular autophagy in health and disease. Basic Res. Cardiol. 115:41. doi: 10.1007/s00395-020-0802-6

Jiang, H., Jia, D., Zhang, B., Yang, W., Dong, Z., Sun, X., et al. (2020). Exercise improves cardiac function and glucose metabolism in mice with experimental myocardial infarction through inhibiting HDAC4 and upregulating GLUT1 expression. Basic Res. Cardiol. 115:28. doi: 10.1007/s00395-020-0787-1

Jiang, J., Chang, X., Nie, Y., Shen, Y., Liang, X., Peng, Y., et al. (2021). Peripheral administration of a cell-penetrating MOTS-c analogue enhances memory and attenuates $\mathrm{A} \beta(1-42)$ - or LPS-Induced memory impairment through inhibiting neuroinflammation. ACS Chem. Neurosci. 12, 1506-1518. doi: 10. 1021/acschemneuro.0c00782

Jin, J. Y., Wei, X. X., Zhi, X. L., Wang, X. H., and Meng, D. (2021). Drp1dependent mitochondrial fission in cardiovascular disease. Acta Pharmacol. Sin. 42, 655-664. doi: 10.1038/s41401-020-00518-y

Jin, Q., Li, R., Hu, N., Xin, T., Zhu, P., Hu, S., et al. (2018). DUSP1 alleviates cardiac ischemia/reperfusion injury by suppressing the Mff-required mitochondrial fission and Bnip3-related mitophagy via the JNK pathways. Redox Biol. 14, 576-587. doi: 10.1016/j.redox.2017.11.004

Jung, J. E., Sun, G., Bautista Garrido, J., Obertas, L., Mobley, A, S., Ting, S. M., et al (2020). The mitochondria-derived peptide humanin improves recovery from intracerebral hemorrhage: implication of mitochondria transfer and microglia phenotype change. J. Neurosci. 40, 2154-2165 doi: 10.1523/jneurosei.2212-19. 2020

Jusic, A., and Devaux, Y. (2020). Mitochondrial noncoding RNA-regulatory network in cardiovascular disease. Basic Res. Cardiol. 115:23. doi: 10.1007/ s00395-020-0783-5

Khabbaz, K. R., Zankoul, F., and Warner, K. G. (2001). Intraoperative metabolic monitoring of the heart: II. Online measurement of myocardial tissue pH. Ann. Thorac. Surg. 72, S2227-S2233. doi: 10.1016/s0003-4975(01)03 284-2

Kim, S. J., Miller, B., Kumagai, H., Silverstein, A. R., Flores, M., and Yen, K. (2021). Mitochondrial-derived peptides in aging and age-related diseases. Geroscience 43, 1113-1121. doi: 10.1007/s11357-020-00262-5

Kim, S. J., Xiao, J., Wan, J., Cohen, P., and Yen, K. (2017). Mitochondrially derived peptides as novel regulators of metabolism. J. Physiol. 595, 6613-6621. doi: $10.1113 /$ jp274472

Klein, L. E., Cui, L., Gong, Z., Su, K., and Muzumdar, R. (2013). A humanin analog decreases oxidative stress and preserves mitochondrial integrity in cardiac myoblasts. Biochem. Biophys. Res. Commun. 440, 197-203. doi: 10.1016/j.bbrc. 2013.08.055

Kleinbongard, P. (2020). Cardioprotection by early metoprolol- attenuation of ischemic vs. reperfusion injury? Basic Res. Cardiol. 115:54. doi: 10.1007/s00395020-0814-2

Krejcova, G., Patocka, J., and Slaninova, J. (2004). Effect of humanin analogues on experimentally induced impairment of spatial memory in rats. J. Pept. Sci. 10, 636-639. doi: 10.1002/psc.569
Kuliawat, R., Klein, L., Gong, Z., Nicoletta-Gentile, M., Nemkal, A., Cui, L., et al. (2013). Potent humanin analog increases glucose-stimulated insulin secretion through enhanced metabolism in the $\beta$ cell. FASEB J. 27, 4890-4898. doi: 10.1096/fj.13-231092

Lahiri, S. K., Quick, A. P., Samson-Couterie, B., Hulsurkar, M., Elzenaar, I., van Oort, R. J., et al. (2020). Nuclear localization of a novel calpain-2 mediated junctophilin-2 C-terminal cleavage peptide promotes cardiomyocyte remodeling. Basic Res. Cardiol. 115:49. doi: 10.1007/s00395-020-0807-1

Lee, C., Kim, K. H., and Cohen, P. (2016). MOTS-c: a novel mitochondrial-derived peptide regulating muscle and fat metabolism. Free Radic. Biol. Med. 100, 182-187. doi: 10.1016/j.freeradbiomed.2016.05.015

Lee, C., Yen, K., and Cohen, P. (2013). Humanin: a harbinger of mitochondrialderived peptides? Trends Endocrinol. 24, 222-228. doi: 10.1016/j.tem.2013. 01.005

Lee, C., Zeng, J., Drew, B. G., Sallam, T., Martin-Montalvo, A., Wan, J., et al. (2015). The mitochondrial-derived peptide MOTS-c promotes metabolic homeostasis and reduces obesity and insulin resistance. Cell Metab. 21, 443-454. doi: 10. 1016/j.cmet.2015.02.009

Li, H., Ren, K., Jiang, T., and Zhao, G. J. (2018). MOTS-c attenuates endothelial dysfunction via suppressing the MAPK/NF-кB pathway Int. J. Cardiol. 268:40. doi: 10.1016/j.ijcard.2018.03.031

Lindner, M., Mehel, H., David, A., Leroy, C., Burtin, M., Friedlander, G., et al. (2020). Fibroblast growth factor 23 decreases PDE4 expression in heart increasing the risk of cardiac arrhythmia. Klotho opposes these effects. Basic Res. Cardiol. 115:51. doi: 10.1

Lobo-Gonzalez, M., Galán-Arriola, C. Rossello, X. González-Del-Hoyo, M., Vilchez, J. P., Higuero Verdejo, M I., et al. (2020). Metoprolol blunts the timedependent progression of infarct size. Basic Res. Cardiol. 115:55. doi: 10.1007/ s00395-020-0812-4

Lu, H., Tang, S., Xue, C., Liu, Y., Wang, J., Zhang, W., et al. (2019). MitochondrialDerived Peptide MOTS-c increases adipose thermogenic activation to promote cold adaptation. Int.J. Mol. Sci. 20:2456. doi: 10.3390/ijms20102456

Lu, X., He, Y., Tang, C., Wang, X., Que, L., Zhu, G., et al. (2020). Triad3A attenuates pathological cardiac hypertrophy involving the augmentation of ubiquitination-mediated degradation of TLR4 and TLR9. Basic Res. Cardiol. 115:19. doi: 10.1007/s00395-020-0779-1

Luciano, F. Zhai, D., Zhu, X., Bailly-Maitre, B., Ricci, J. E., Satterthwait, A. C., et al. (2005). Cytoprotective peptide humanin binds and inhibits proapoptotic Bcl2/Bax family protein BimEL. J. Biol. Chem. 280, 15825-15835. doi: 10.1074/jbc. M413062200

Lyu, J., Wang, M., Kang, X., Xu, H., Cao, Z., Yu, T., et al. (2020). Macrophagemediated regulation of catecholamines in sympathetic neural remodeling after myocardial infarction. Basic Res. Cardiol. 115:56. doi: 10.1007/s00395-0200813-3

Lyu, Y., Jiang, X., and Dai, W. (2015). The roles of a novel inflammatory neopterin in subjects with coronary atherosclerotic heart disease. Int. Immunopharmacol. 24, 169-172. doi: 10.1016/j.intimp.2014.11.013

Ma, Z. W., and Liu, D. X. (2018). Humanin decreases mitochondrial membrane permeability by inhibiting the membrane association and oligomerization of Bax and Bid proteins. Acta Pharmacol. Sin. 39, 1012-1021. doi: 10.1038/aps. 2017.169

Mangalhara, K. C., and Shadel, G. S. (2018). A mitochondrial-derived peptide exercises the nuclear option. Cell Metab. 28, 330-331. doi: 10.1016/j.cmet.2018. 08.017

McCully, J. D., Rousou, A. J., Parker, R. A., and Levitsky, S. (2007). Age- and gender-related differences in mitochondrial oxygen consumption and calcium with cardioplegia and diazoxide. Ann. Thorac. Surg. 83, 1102-1109. doi: 10. 1016/j.athoracsur.2006.10.059

Mehta, H. H., Xiao, J., Ramirez, R., Miller, B., Kim, S. J., Cohen, P., et al. (2019). Metabolomic profile of diet-induced obesity mice in response to humanin and small humanin-like peptide 2 treatment. Metabolomics 15:88. doi: 10.1007/ s11306-019-1549-7

Michel, S., Canonne, M., Arnould, T., and Renard, P. (2015). Inhibition of mitochondrial genome expression triggers the activation of CHOP-10 by a cell signaling dependent on the integrated stress response but not the mitochondrial unfolded protein response. Mitochondrion 21, 58-68. doi: 10.1016/j.mito.2015. 01.005 
Ming, W., Lu, G., Xin, S., Huanyu, L., Yinghao, J., Xiaoying, L., et al. (2016). Mitochondria related peptide MOTS-c suppresses ovariectomy-induced bone loss via AMPK activation. Biochem. Biophys. Res. Commun. 476, 412-419. doi: 10.1016/j.bbrc.2016.05.135

Mottis, A., Herzig, S., and Auwerx, J. (2019). Mitocellular communication: shaping health and disease. Science 366, 827-832. doi: 10.1126/science.aax3768

Muzumdar, R. H., Huffman, D. M., Atzmon, G., Buettner, C., Cobb, L. J., Fishman, S., et al. (2009). Humanin: a novel central regulator of peripheral insulin action. PLoS One 4:e6334. doi: 10.1371/journal.pone.0006334

Muzumdar, R. H., Huffman, D. M., Calvert, J. W., Jha, S., Weinberg, Y., Cui, L., et al. (2010). Acute humanin therapy attenuates myocardial ischemia and reperfusion injury in mice. Arterioscler. Thromb. Vasc. Biol. 30, 1940-1948. doi: 10.1161/atvbaha.110.205997

Olivetti, G., Capasso, J. M., Meggs, L. G., Sonnenblick, E. H., and Anversa, P. (1991). Cellular basis of chronic ventricular remodeling after myocardial infarction in rats. Circ. Res. 68, 856-869. doi: 10.1161/01.res.68.3.856

Ollauri-Ibáñez, C., Núñez-Gómez, E., Egido-Turrión, C., Silva-Sousa, L., DíazRodríguez, E., Rodríguez-Barbero, A., et al. (2020). Continuous endoglin (CD105) overexpression disrupts angiogenesis and facilitates tumor cell metastasis. Angiogenesis 23, 231-247. doi: 10.1007/s10456-019-09703-y

Pacher, P., Nivorozhkin, A., and Szabó, C. (2006). Therapeutic effects of xanthine oxidase inhibitors: renaissance half a century after the discovery of allopurinol. Pharmacol. Rev. 58, 87-114. doi: 10.1124/pr.58.1.6

Pagliaro, P., and Penna, C. (2015). Redox signalling and cardioprotection: translatability and mechanism. Br. J. Pharmacol. 172, 1974-1995. doi: 10.1111/ bph. 12975

Pan, X., Liu, J., Nguyen, T., Liu, C., Sun, J., Teng, Y., et al. (2013). The physiological role of mitochondrial calcium revealed by mice lacking the mitochondrial calcium uniporter. Nat. Cell Biol. 15, 1464-1472. doi: 10.1038/ncb2868

Qiao, K., Liu, Y., Xu, Z., Zhang, H., Zhang, H., Zhang, C., et al. (2021). RNA m6A methylation promotes the formation of vasculogenic mimicry in hepatocellular carcinoma via Hippo pathway. Angiogenesis 24, 83-96. doi: 10.1007/s10456020-09744-8

Qin, Q., Delrio, S., Wan, J., Jay Widmer, R., Cohen, P., Lerman, L. O., et al. (2018a). Downregulation of circulating MOTS-c levels in patients with coronary endothelial dysfunction. Int. J. Cardiol. 254, 23-27. doi: 10.1016/j.ijcard.2017. 12.001

Qin, Q., Jin, J., He, F., Zheng, Y., Li, T., Zhang, Y., et al. (2018b). Humanin promotes mitochondrial biogenesis in pancreatic MIN6 $\beta$-cells. Biochem. Biophys. Res. Commun. 497, 292-297. doi: 10.1016/

Quirós, P. M., Mottis, A., and Auwerx, J. (2016). . Mitonuclear communication in homeostasis and stress. Nat. Rev. Mol. Cell Biol. 17, 213-226. doi: 10.1038/hrm. 2016.23

Reed, G. W., Rossi, J. E., and Cannon, C. P. (2017). Acute myocardial infarction. Lancet 389, 197-210. doi: 10.1016/s0140-6736(16)30677-8

Reimer, K. A., Lowe, J. E., Rasmussen, M. M., and Jennings, R. B. (1977). The wavefront phenomenon of ischemic cell death. 1. Myocardial infarct size vs duration of coronary occlusion in dogs. Circulation 56, 786-794. doi: 10.1161/ 01.cir.56.5.786

Ren, L., Li, Q., You, T., Zhao, X., Xu, X., Tang, C., et al. (2020). Humanin analogue, HNG, inhibits platelet activation and thrombus formation by stabilizing platelet microtubules. J. Cell. Mol. Med. 24, 4773-4783. doi: 10.1111/jcmm.15151

Ruiz-Meana, M., Abellán, A., Miró-Casas, E., Agulló, E., and Garcia-Dorado, D. (2009). Role of sarcoplasmic reticulum in mitochondrial permeability transition and cardiomyocyte death during reperfusion. Am. J. Physiol. Heart Circ. Physiol. 297, H1281-H1289. doi: 10.1152/ajpheart.00435.2009

Ryan, M. T., and Hoogenraad, N. J. (2007). Mitochondrial-nuclear communications. Annu. Rev. Biochem. 76, 701-722. doi: 10.1146/annurev. biochem.76.052305.091720

Santosa, S. M., Guo, K., Yamakawa, M., Ivakhnitskaia, E., Chawla, N., Nguyen, T., et al. (2020). Simultaneous fluorescence imaging of distinct nerve and blood vessel patterns in dual Thy1-YFP and Flt1-DsRed transgenic mice. Angiogenesis 23, 459-477. doi: 10.1007/s10456-020-09724-y

Sawashita, Y., Hirata, N., Yoshikawa, Y., Terada, H., Tokinaga, Y., and Yamakage, M. (2020). Remote ischemic preconditioning reduces myocardial ischemiareperfusion injury through unacylated ghrelin-induced activation of the JAK/STAT pathway. Basic Res. Cardiol. 115:50. doi: 10.1007/s00395-0200809-Z
Schinner, C., Olivares-Florez, S., Schlipp, A., Trenz, S., Feinendegen, M., Flaswinkel, H., et al. (2020). The inotropic agent digitoxin strengthens desmosomal adhesion in cardiac myocytes in an ERK1/2-dependent manner. Basic Res. Cardiol. 115:46. doi: 10.1007/s00395-020-0805-3

Seano, G., and Jain, R. K. (2020). Vessel co-option in glioblastoma: emerging insights and opportunities. Angiogenesis 23, 9-16. doi: 10.1007/s10456-01909691-z

Selvaraju, V., Thirunavukkarasu, M., Joshi, M., Oriowo, B., Shaikh, I. A., Rishi, M. T., et al. (2020). Deletion of newly described pro-survival molecule Pellino-1 increases oxidative stress, downregulates cIAP2/NF- $\kappa$ B cell survival pathway, reduces angiogenic response, and thereby aggravates tissue function in mouse ischemic models. Basic Res. Cardiol. 115:45. doi: 10.1007/s00395-020-0 804-4

Singh, E., Redgrave, R. E., Phillips, H. M., and Arthur, H. M. (2020). Arterial endoglin does not protect against arteriovenous malformations. Angiogenesis 23, 559-566. doi: 10.1007/s10456-020-09731-z

Smadja, D. M., Guerin, C. L., Chocron, R., Yatim, N., Boussier, J., Gendron, N., et al. (2020). Angiopoietin-2 as a marker of endothelial activation is a good predictor factor for intensive care unit admission of COVID-19 patients. Angiogenesis 23, 611-620. doi: 10.1007/s10456-020-09730-0

Sommer, S., Leistner, M., Aleksic, I., Schimmer C., Alhussini, K., Kanofsky, P., et al. (2016). Impact of levosimendan and ischaemia-reperfusion injury on myocardial subsarcolemmal mitochondrial respiratory chain, mitochondrial membrane potential, Ca2+ cycling and ATP synthesis. Eur. J. Cardiothorac. Surg. 49, e54-e62. doi: 10.1093/ejcts/ezv397

Sreekumar, P. G., Ishikawa, K. Spee, C., Mehta,H.H., Wan, J., Yen, K., et al. (2016). The mitochondrial-derived peptide humanin protects RPE cells from oxidative stress, senescence, and mitochondrial dysfunction. Invest. Ophthalmol. Vis. Sci. $57,1238-1253$.

Sreekumar, P. G., and Kannan, R. (2020). Mechanisms of protection of retinal pigment epithelial cells from oxidant injury by humanin and other mitochondrial-derived peptides: implications for age-related macular degeneration. Redox Biol. 37:101663. doi: 10.1016/j.redox.2020. 101663

eenman, M., and I ande, G. (2017). Cardiac aging and heart disease in humans. Biophys. Rev. 9, 131-137. doi: 10.1007/s12551-017-0255-9

Steínberg, G.R., and Kemp, B. E. (2009). AMPK in health and disease. Physiol. Rev. 89, 1025-1078. doi: 10.1152/physrev.00011.2008

árez-Rivero, J. M., Villanueva-Paz, M., de la Cruz-Ojeda, P., de la Mata, M., Cotán, D., Oropesa-Ávila, M., et al. (2016). Mitochondrial dynamics in mitochondrial diseases. Diseases 5:1. doi: 10.3390/diseases501 0001

Sutton, M. G., and Sharpe, N. (2000). Left ventricular remodeling after myocardial infarction: pathophysiology and therapy. Circulation 101, 2981-2988. doi: 10. 1161/01.cir.101.25.2981

Szulcek, R., Sanchez-Duffhues, G., Rol, N., Pan, X., Tsonaka, R., Dickhoff, C., et al. (2020). Exacerbated inflammatory signaling underlies aberrant response to BMP9 in pulmonary arterial hypertension lung endothelial cells. Angiogenesis 23, 699-714. doi: 10.1007/s10456-020-09741-x

Tabas, I., Williams, K. J., and Borén, J. (2007). Subendothelial lipoprotein retention as the initiating process in atherosclerosis: update and therapeutic implications. Circulation 116, 1832-1844.

Tajima, H., Kawasumi, M., Chiba, T., Yamada, M., Yamashita, K., Nawa, M., et al. (2005). A humanin derivative, S14G-HN, prevents amyloid-beta-induced memory impairment in mice. J. Neurosci. Res. 79, 714-723. doi: 10.1002/jnr. 20391

Tan, Y., Mui, D., Toan, S., Zhu, P., Li, R., and Zhou, H. (2020). SERCA overexpression improves mitochondrial quality control and attenuates cardiac microvascular ischemia-reperfusion injury. Ther. Nucleic Acids 22, 696-707. doi: 10.1016/j.omtn.2020.09.013

Tennant, R., and Wiggers, C. J. (1935). The effect of coronary occlusion on myocardial contraction. Am. J. Physiol. Legacy Content 112, 351-361. doi: 10. 1152/ajplegacy.1935.112.2.351

Thummasorn, S., Shinlapawittayatorn, K., Chattipakorn, S. C., and Chattipakorn, N. (2017). High-dose Humanin analogue applied during ischemia exerts cardioprotection against ischemia/reperfusion injury by reducing mitochondrial dysfunction. Cardiovasc. Ther. 35:e12289. doi: $10.1111 / 1755-5922.12289$ 
Thummasorn, S., Shinlapawittayatorn, K., Khamseekaew, J., Jaiwongkam, T., Chattipakorn, S. C., and Chattipakorn, N. (2018). Humanin directly protects cardiac mitochondria against dysfunction initiated by oxidative stress by decreasing complex I activity. Mitochondrion 38, 31-40. doi: 10.1016/j.mito. 2017.08.001

Tomita, Y., Cakir, B., Liu, C. H., Fu, Z., Huang, S., Cho, S. S., et al. (2020). Free fatty acid receptor 4 activation protects against choroidal neovascularization in mice. Angiogenesis 23, 385-394. doi: 10.1007/s10456-020-09717-x

Walker, C. A., Crawford, F. A. Jr., and Spinale, F. G. (2000). Myocyte contractile dysfunction with hypertrophy and failure: relevance to cardiac surgery. J. Thorac. Cardiovasc. Surg. 119, 388-400. doi: 10.1016/s0022-5223(00)70199-1

Wang, J., Toan, S., Li, R., and Zhou, H. (2020a). Melatonin fine-tunes intracellular calcium signals and eliminates myocardial damage through the IP3R/MCU pathways in cardiorenal syndrome type 3. Biochem. Pharmacol. 174:113832. doi: $10.1016 /$ j.bcp. 2020.113832

Wang, J., Toan, S., and Zhou, H. (2020b). New insights into the role of mitochondria in cardiac microvascular ischemia/reperfusion injury. Angiogenesis 23, 299-314. doi: 10.1007/s10456-020-09720-2

Wang, J., Zhu, P., Li, R., Ren, J., Zhang, Y., and Zhou, H. (2020c). Bax inhibitor 1 preserves mitochondrial homeostasis in acute kidney injury through promoting mitochondrial retention of PHB2. Theranostics 10, 384-397. doi: 10.7150/thno. 40098

Wang, J., Zhu, P., Toan, S., Li, R., Ren, J., and Zhou, H. (2020d). Pum2-Mff axis fine-tunes mitochondrial quality control in acute ischemic kidney injury. Cell Biol. Toxicol. 36, 365-378. doi: 10.1007/s10565-020-09513-9

Wang, Z., Lu, Y. L., Zhao, W. T., Zhong, J., Lin, X., Sun, Z., et al. (2020e). Distinct origins and functions of cardiac orthotopic macrophages. Basic Res. Cardiol. 115:8. doi: 10.1007/s00395-019-0769-3

Wang, X., Wu, Z., He, Y., Zhang, H., Tian, L., Zheng, C., et al. (2018). Humanin prevents high glucose-induced monocyte adhesion to endothelial cells by targeting KLF2. Mol. Immunol. 101, 245-250. doi: 10.1016/j.molimm.2018. 07.008

Watanabe, E., Wada, T., Okekawa, A., Kitamura, F., Komatsu, G., Onogi, Y., et al. (2020). Stromal cell-derived factor 1 (SDF1) attenuates platelet-derived growth factor-B (PDGF-B)-induced vascular remodeling for adipose tissue expansion in obesity. Angiogenesis 23, 667-684. doi: 10.1007/s10456-020-09738-6

Watson, S. A., Dendorfer, A., Thum, T., and Perbellini, F. (2020). A practical guide for investigating cardiac physiology using living myocardial slices. Basic Res. Cardiol. 115:61. doi: 10.1007/s00395-020-00822-y

Wei, M., Gan, L., Liu, Z., Liu, L., Chang, J. R., Mitochondrial-Derived Peptide MOTS-c attenuates vascular calcification and secondary myocardial remodeling via adenøsine monophosphate-activated protein kinase signaling pathway. Cardiorenal Med. 10, 42-50. doi. 10.1159/ 000503224

Weng, F. B., Zhu, L. F., Zhou, J. X., Shan, Y., Tian, Z. G., and Yang, L. W. (2021). MOTS-c accelerates bone fracture healing by stimulating osteogenesis of bone marrow mesenchymal stem cells via positively regulating FOXF1 to activate the TGF- $\beta$ pathway. Eur. Rev. Med. Pharmacol. Sci. 25:2459. doi: 10.26355/eurrev_ 202103_25396

Wijenayake, S., and Storey, K B. (2021). Oxidative Damage? Not a Problem! The characterization of humanin-like mitochondrial peptide in anoxia tolerant freshwater turtles. Protein J. 40, 87-107. doi: 10.1007/s10930-020-09944-7

Wincewicz, A., and Woltanowski, P. (2020). Leopold Auerbach's achievements in the field of vascular system. Angiogenesis 23, 577-579. doi: 10.1007/s10456-02009739-5

Winter, M. P., Sharma, S., Altmann, J., Seidl, V., Panzenböck, A., Alimohammadi, A., et al. (2020). Interruption of vascular endothelial growth factor receptor 2 signaling induces a proliferative pulmonary vasculopathy and pulmonary hypertension. Basic Res. Cardiol. 115:58. doi: 10.1007/s00395-020-0811-5

Wischmann, P., Kuhn, V., Suvorava, T., Muessig, J. M., Fischer, J. W., Isakson, B. E., et al. (2020). Anaemia is associated with severe RBC dysfunction and a reduced circulating NO pool: vascular and cardiac eNOS are crucial for the adaptation to anaemia. Basic Res. Cardiol. 115:43. doi: 10.1007/s00395-020-0799-x

Xiao, J., Howard, L., Wan, J., Wiggins, E., Vidal, A., Cohen, P., et al. (2017). Low circulating levels of the mitochondrial-peptide hormone SHLP2: novel biomarker for prostate cancer risk. Oncotarget 8, 94900-94909. doi: 10.18632/ oncotarget. 20134
Xie, Y., Liu, Z. H., Li, X. Y., Zhou, Y. D., Xu, X., Hu, L. F., et al. (2014). Protection effect of [Gly14]-Humanin from apoptosis induced by high glucose in human umbilical vein endothelial cells. Diabetes Res. Clin. Pract. 106, 560-566. doi: 10.1016/j.diabres.2014.09.020

Yan, Z., Zhu, S., Wang, H., Wang, L., Du, T., Ye, Z., et al. (2019). MOTS-c inhibits Osteolysis in the Mouse Calvaria by affecting osteocyte-osteoclast crosstalk and inhibiting inflammation. Pharmacol. Res. 147:104381. doi: 10.1016/j.phrs.2019. 104381

Yang, Q. K., Chen, T., Wang, S. Q., Zhang, X. J., and Yao, Z. X. (2020). Apatinib as targeted therapy for advanced bone and soft tissue sarcoma: a dilemma of reversing multidrug resistance while suffering drug resistance itself. Angiogenesis 23, 279-298. doi: 10.1007/s10456-020-09716-y

Yang, Y., Gao, H., Zhou, H., Liu, Q., Qi, Z., Zhang, Y., et al. (2019). The role of mitochondria-derived peptides in cardiovascular disease: recent updates. Biomed. Pharmacother. 117:109075. doi: 10.1016/j.biopha.2019.109075

Yang, Y., Ma, L., Wang, C., Song, M., Li, C., Chen, M., et al. (2020). Matrix metalloproteinase-7 in platelet-activated macrophages accounts for cardiac remodeling in uremic mice. Basic Res. Cardiol. 115:30. doi: 10.1007/s00395020-0789-z

Yen, K., Lee, C., Mehta, H., and Cohen, P. (2013) The emerging role of the mitochondrial-derived peptide humanin in stress resistance.J. Mol. Endocrinol. 50, R11-R19. doi: 10.1530/jme-12-0203

Zapała, B., Kaczyński, Ł., Kieć-Wilk, B. Staszel, T., Knapp, A., Thoresen, G. H., et al. (2010). Humanins, the neuroprotective and cytoprotective peptides with antiapoptotic and anti-inflammatory properties. Pharmacol. Rep. 62, 767-777. doi: 10.1016/s1734-1140(10)703

Zhai, D., Ye, Z., Jiang, Y., Xu, O., Ruan, B. Yang, Y., etal. (2017). MOTS-c peptide increases survival and decreases bacterial load in mice infected with MRSA. Mol. Immunol. 92, 151-160. doi: 10.1016/j.molimm.2017.10.017

Zhang, L. Zhu, X. Y., Zhao, Y. Eirin, A., Liu, L., Ferguson, C. M., et al. (2020). Selective intrarenal delivery of mesenchymal stem cell-derived extracellular vesicles attenuates myocardial injury in experimental metabolic renovascular disease. Basic Res. Cardiol. 115:16. doi: 10.1007/s00395-019-0772-8

Zhang, Y., Wang, S., and Dudley, A. C. (2020). Models and molecular mechanisms of blood vessel co-option by cancer cells. Angiogenesis 23, 17-25. doi: 10.1007/ s10456-019-09684-y

Zhao, Q., Molina-Portela, M. D. P., Parveen, A., Adler, A., Adler, C., E, H., et al. (2020). Heterogeneity and chimerism of endothelial cells revealed by singlecell transcriptome in orthotopic liver tumors. Angiogenesis 23, 581-597. doi: 10.1007/s10456-020-09727-9

Zhao, S. T., Zhao, L., and Li, J. H. (2013). Neuroprotective Peptide humanin inhibits inflammatory response in astrocytes induced by lipopolysaccharide. Neurochem. Res. 38, 581-588. doi: 10.1007/s11064-012-0951-6

Zhou, H., Li, D., Zhu, P., Hu, S., Hu, N., Ma, S., et al. (2017a). Melatonin suppresses platelet activation and function against cardiac ischemia/reperfusion injury via PPARgamma/FUNDC1/mitophagy pathways. J. Pineal Res. 63:e12438. doi: $10.1111 /$ jpi.12438

Zhou, H., Zhang, Y., Hu, S., Shi, C., Zhu, P., Ma, Q., et al. (2017b). Melatonin protects cardiac microvasculature against ischemia/reperfusion injury via suppression of mitochondrial fission-VDAC1-HK2-mPTP-mitophagy axis. J. Pineal Res. 63:e12413. doi: 10.1111/jpi.12413

Zhou, H., Shi, C., Hu, S., Zhu, H., Ren, J., and Chen, Y. (2018a). BI1 is associated with microvascular protection in cardiac ischemia reperfusion injury via repressing Syk-Nox2-Drp1-mitochondrial fission pathways. Angiogenesis 21, 599-615. doi: 10.1007/s10456-018-9611-z

Zhou, H., Wang, J., Zhu, P., Zhu, H., Toan, S., Hu, S., et al. (2018b). NR4A1 aggravates the cardiac microvascular ischemia reperfusion injury through suppressing FUNDC1-mediated mitophagy and promoting Mffrequired mitochondrial fission by CK2alpha. Basic Res. Cardiol. 113:23. doi: 10.1007/s00395-018-0682-1

Zhou, H., Wang, S., Hu, S., Chen, Y., and Ren, J. (2018c). ER-mitochondria microdomains in cardiac ischemia-reperfusion injury: a fresh perspective. Front. Physiol. 9:755. doi: 10.3389/fphys.2018.00755

Zhou, H., Toan, S., Zhu, P., Wang, J., Ren, J., and Zhang, Y. (2020). DNAPKcs promotes cardiac ischemia reperfusion injury through mitigating BI-1governed mitochondrial homeostasis. Basic Res. Cardiol. 115:11. doi: 10.1007/ s00395-019-0773-7 
Zhou, H., Zhu, P., Wang, J., Toan, S., and Ren, J. (2019). DNA-PKcs promotes alcohol-related liver disease by activating Drp1-related mitochondrial fission and repressing FUNDC1-required mitophagy. Signal Transduct. Target. Ther. 4:56. doi: 10.1038/s41392-019-0094-1

Zhu, H., Tan, Y., Du, W., Li, Y., Toan, S., Mui, D., et al. (2021). Phosphoglycerate mutase 5 exacerbates cardiac ischemia-reperfusion injury through disrupting mitochondrial quality control. Redox Biol. 38:101777. doi: 10.1016/j.redox.2020. 101777

Zhu, P., Hu, S., Jin, Q., Li, D., Tian, F., Toan, S., et al. (2018). Ripk3 promotes ER stress-induced necroptosis in cardiac IR injury: a mechanism involving calcium overload/XO/ROS/mPTP pathway. Redox Biol. 16, 157-168. doi: 10. 1016/j.redox.2018.02.019

Zhu, W. W., Wang, S. R., Liu, Z. H., Cao, Y. J., Wang, F., Wang, J., et al. (2017). Gly[14]-humanin inhibits ox-LDL uptake and stimulates cholesterol efflux in macrophage-derived foam cells. Biochem. Biophys. Res. Commun. 482, 93-99. doi: $10.1016 /$ j.bbrc. 2016.10 .138
Conflict of Interest: The authors declare that the research was conducted in the absence of any commercial or financial relationships that could be construed as a potential conflict of interest.

Publisher's Note: All claims expressed in this article are solely those of the authors and do not necessarily represent those of their affiliated organizations, or those of the publisher, the editors and the reviewers. Any product that may be evaluated in this article, or claim that may be made by its manufacturer, is not guaranteed or endorsed by the publisher.

Copyright (C) $2021 \mathrm{Wu}$, Kampmann and Qian. This is an open-access article distributed under the terms of the Creative Commons Attribution License (CC BY). The use, distribution or reproduction in other forums is permitted, provided the original author(s) and the copyright owner(s) are credited and that the original publication in this journal is cited, in accordance with accepted academic practice. No use, distribution or reproduction is permitted which does not comply with these terms.

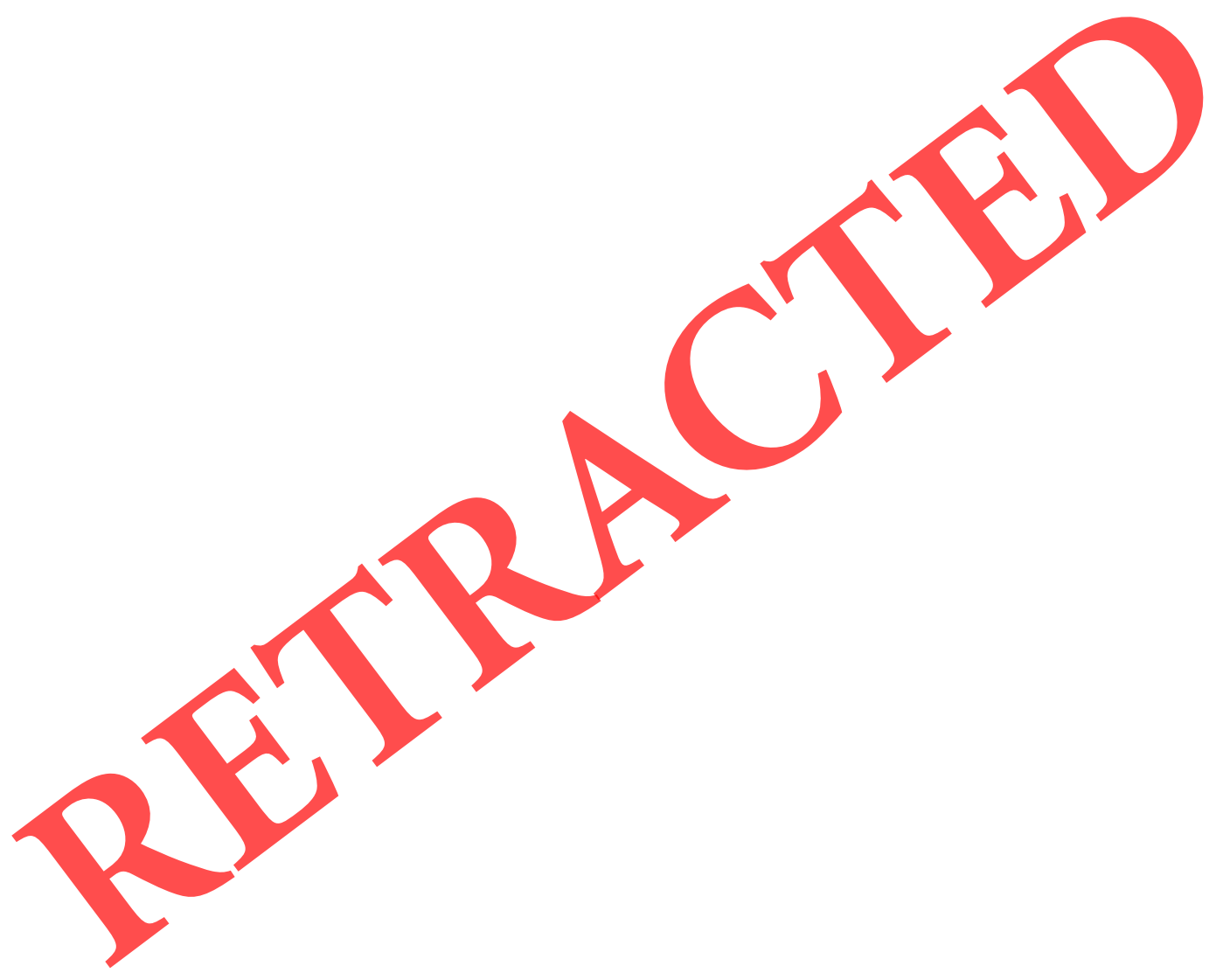

